\title{
Analysis of Genetic Algorithm and Particle Swarm Optimization for Warehouse with Supply Chain Management in Inventory Control
}

\author{
Ajay Singh Yadav \\ Ph.D., Assistant Professor \\ SRM University, NCR, \\ Campus, \\ Ghaziabad, U.P.
}

\author{
Prerna Maheshwari \\ (Sharma) \\ Ph.D., Assistant Professor \\ SRM University, NCR, \\ Campus,
}

\author{
Ghaziabad, U.P \\ Anupam Swami \\ Assistant Professor \\ Govt. P.G. College, \\ Sambhal, U.P.
}

\begin{abstract}
The purpose of the proposed study is to give a new dimension on warehouse with Particle Swarm Optimization and Economic Load Dispatch method using genetic algorithm processes in supply chain in inventory optimization to describe the certain and uncertain market demand which is based on supply reliability and to develop more realistic and more flexible models. we hope that the proposed study has a great potential to solve various practical tribulations related to the warehouse with Particle Swarm Optimization and Economic Load Dispatch method using genetic algorithm processes in supply chain in inventory optimization and also provide a general review for the application of soft computing techniques like genetic algorithms to use for improve the effectiveness and efficiency for various aspect of warehouse with Particle Swarm Optimization and Economic Load Dispatch method control using genetic algorithm.
\end{abstract}

\section{Keywords}

Particle Swarm Optimization, genetic algorithm, warehouse, Supply Chain management, Inventory control.

\section{INTRODUCTION}

\subsection{Inventory control}

Inventory control, otherwise known as stock control, is used to show how much stock have to maid available at any time, and how tracks are kept for it. It applies to every item that uses to produce a product or service, from raw materials to finished goods. It covers stock at every stage of the production process, from purchase and delivery to using and re-ordering the stock. Efficient stock control allows an organization/industry/company to have the right amount of stock in the right place at the right time. It ensures that capital is not tied up unnecessarily, and protects production if problems arise with the supply chain. Inventory control is the techniques of maintaining stock-items at desired levels. The purpose of all inventory models is to minimize inventory costs. As a result of the inventory model, a designer of aircondition machine decided to redesign its old model machine to enhance its working efficiency and reduce inventory costs in meeting a global market for its air-condition machines.

Inventory is held throughout the supply chain in the form of raw materials, work in process and finished goods. Inventory exists in the supply chain because of a mismatch between supply and demand. This mismatch is intentional at a manufacturer, where it is economical to manufacture in large lots that are then stored for future sales. The mismatch is also intentional at a retail store where inventory is held in anticipation of future demand.
Inventory is a major source of cost in a supply chain and has a huge impact on responsiveness. An important role that inventory plays in the supply chain is (1) to increase the amount of demand that can be satisfied by having the product ready and available when the customer wants it. (2) To reduce cost by exploiting economics of scale that may exist during production and distribution. (3) To support a firm's competitive strategy. If a firm's competitive strategy requires very high level of responsiveness, a company can achieve this responsiveness by locating large amounts of inventory close to a customer. Conversely, a company can also use inventory to become more efficient by reducing inventory through centralized stocking.

We use a variety of food in our daily life to eat. Some of us may take rice, while others may take wheat in the form of different dishes as our main food vegetables are sometime available easily when we demand and sometime not, but can be obtained with the payment of more cost than during normal availability. But have we ever thought that from where vegetables, the rice paddy or wheat from which these food items are prepared comes. We all know that these food grains are not produced throughout the year but we need to eat them every day throughout the year. So, how are the farmers able to supply these continuously to us? We might be thinking that they store the food grains in a proper place and supply them at the time of need. Yes, we are right. Since the production takes place during a particular season and in specific areas, there is a need to store these food grains systematically. In our home we may keep limited stock for our own consumption e.g. 4-5 $\mathrm{kg}$ of pulses, $20-50 \mathrm{~kg}$ of wheat/rice and we need to buy it again form somewhere(shops) after an interval of time. For this purpose i.e. to provide us the desired items when we needed, there are certain places or stores, where these items are stored in huge quantities in a proper and systematic way. We need different types of goods in our day-to-day life. We may buy some of these items in bulk and store them in our house. Similarly, businessmen also need a variety of goods for the business but some of them may not be available all the time. But, they need those items throughout the year without any break. For example, in electricity power plant (thermal plant) needed coal through the year for the production of electricity, coal is used as raw material, coal is not available at every place and is to be transported from a particular place which takes time so the power plant needed to store it for the year to use when it required. Since the demand for coal in the Producer is continuous process for the production of electricity. Thus, the need for storage arises both for raw material as well as finished products (i.e. electricity). Since storage involves proper arrangement for preserving goods from the time of their production or purchase till the actual 
use therefore it require proper space for storage. When this storage is done on a large scale and in a specified manner it is called 'Warehousing'.

\subsection{Genetic Algorithm}

The principles of Genetic Algorithms (GA) and the mathematical framework underlying it were developed in the late 1960s (Holland, 1962; Kristinson and Dumont, 1992; Koppen et al., 2006). GA is normally discussed in the context of Evolutionary Computing (EC). The core methodologies of EC are Genetic Algorithms (GA), Evolutionary Programming (EP), Evolution Strategies (ES) and Genetic Programming (GP) (Oduguwa et al., 2005). In GA, attempt is made to model the processes underlying population genetic theory by using random search. GAs uses the survival-of-the-fittest strategy, where stronger individuals in a population have a higher chance of creating an offspring. To achieve this, the current input (population) is used to create a new and better population based on specified constraints. The inputs are normally represented as string and they model chromosome in human genetics. In materials engineering, for example, the input string will represent some properties of materials that are of interest.

one iteration of the algorithm is referred to as a generation. The basic GA is very generic and there are many aspects that can be implemented differently according to the problem ( for instance, representation of solution or chromosomes, type of encoding, selection strategy, type of crossover and mutation operators, etc. ) in practice, GA are implemented by having arrays of bits or characters to represent the chromosomes. The individuals in the population then go through a process of simulated evolution. Simple bit manipulation operations allow the implementation of crossover, mutation and other operations. The number of bits for every gene (parameter) and the decimal range in which they decode are usually the same but precludes the utilization of a different number of bits or range

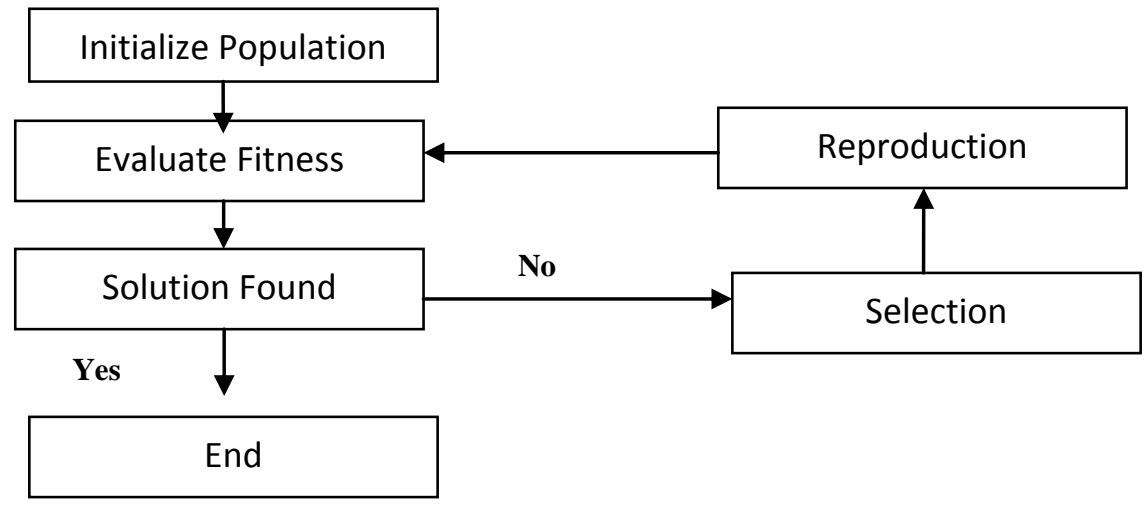

Flow chart of basic genetic algorithm

When compared to other evolutionary algorithms one of the most important GA feature is its focus on fixed length character strings although variable length strings and other structures have been used.

\section{Step 1: Start}

(Randomly generate population of $\mathrm{n}$ chromosomes as per population size.)

\section{Step 2: Fitness}

(Evaluate the fitness $f(y)$ of each chromosome $y$ in the population)

\section{Step 3: New population}

(Create new population by repeating following steps until the new population is complete.)

\section{a. Selection}

(Select two parent chromosomes from a population.)

\section{b. Crossover}

(With a crossover probability, crossover the parents to form a new offspring. If no crossover was performed, offspring is the exact copy of parents.)

\section{c. Mutation}

(With a mutation probability, mutate new offspring at each locus.)

\section{d. Accepting}

(Place new offspring in the new population.)

\section{Step 4: Replace}

(Use new generated population for a future run of the algorithm.)

\section{Step 5: Test}

(If the end condition is satisfied, stop, and return the best solution in current population.)

\section{Step 6: Loop}

Go to step 2

Step 7: Stop

(Stop when the fittest value is obtained.)

We are using those basic steps for finding the optimal resources for an organization in Medium range prospective using MATLAB software package

\subsection{Particle Swarm Optimization}

Particle swarm optimization is initialized by a population of random solution and each potential solution is assigned a randomized velocity. The potential solutions called particles are then flown through the problem space. Each particle keeps track of its coordinates in the problem space which are associated with the best solution or fitness achieved so far the fitness value is also stored this value is called pbest. Another best value that is tracked by the global version of the PSO is the overall best value and its location obtained so far by any particle in the population. This value is termed gbest.

Thus at each time step the particle change its velocity and moves towards its pbest and gbest this is the global version of PSO when in addition to pbest each particle keeps track of the 
best solution called nbest or lbest attained within a local topological neighbourhood of the particles the process is known as the local version of PSO

\section{RELATED WORKS}

Narmadha at. al. (2010) proposed inventory management is considered to be an important field in Supply Chain Management because the cost of inventories in a supply chain accounts for about $30 \%$ of the value of the product. The service provided to the customer eventually gets enhanced once the efficient and effective management of inventory is carried out all through the supply chain. The precise estimation of optimal inventory is essential since shortage of inventory yields to lost sales, while excess of inventory may result in pointless storage costs. Thus the determination of the inventory to be held at various levels in a supply chain becomes inevitable so as to ensure minimal cost for the supply chain. The minimization of the total supply chain cost can only be achieved when optimization of the base stock level is carried out at each member of the supply chain. This paper deals with the problem of determination of base-stock levels in a ten member serial supply chain with multiple products produced by factories using Uniform Crossover Genetic Algorithms. The complexity of the problem increases when more distribution centers and agents and multiple products were involved. These considerations leading to very complex inventory management process has been resolved in this work.

Radhakrishnan et. al. (2009) gives a inventory management plays a vital role in supply chain management. The service provided to the customer eventually gets enhanced once the efficient and effective management of inventory is carried out all through the supply chain. Thus the determination of the inventory to be held at various levels in a supply chain becomes inevitable so as to ensure minimal cost for the supply chain. Minimizing the total supply chain cost is meant for minimizing holding and shortage cost in the entire supply chain. The minimization of the total supply chain cost can only be achieved when optimization of the base stock level is carried out at each member of the supply chain. A serious issue in the implementation of the same is that the excess stock level and shortage level is not static for every period. In this paper, we have developed a new and efficient approach that works on Genetic Algorithms in order to distinctively determine the most probable excess stock level and shortage level required for inventory optimization in the supply chain such that the total supply chain cost is minimized.

Singh and Kumar (2011) gives a Optimal inventory control is one of the significant tasks in supply chain management. The optimal inventory control methodologies intend to reduce the supply chain cost by controlling the inventory in an effective manner, such that, the SC members will not be affected by surplus as well as shortage of inventory. In this paper, we propose an efficient approach that effectively utilizes the Genetic Algorithm for optimal inventory control. This paper reports a method based on genetic algorithm to optimize inventory in supply chain management. We focus specifically on determining the most probable excess stock level and shortage level required for inventory optimization in the supply chain so that the total supply chain cost is minimized .We apply our methods on three stage supply chain model studied for optimization

Priya and Iyakutti (2011) presents an approach to optimize the reorder level (ROL) in the manufacturing unit taking consideration of the stock levels at the factory and the distribution centers of the supply chain, which in turn helps the production unit to optimize the production level and minimizing the inventory holding cost. Genetic algorithm is used for the optimization in a multi product, multi level supply chain in a web enabled environment. This prediction of optimal ROL enables the manufacturing unit to overcome the excess/ shortage of stock levels in the upcoming period

Thakur and Desai (2013) a study With the dramatic increase in the use of the Internet for supply chain-related activities, there is a growing need for services that can analyze current and future purchases possibilities as well as current and future demand levels and determine efficient and economical strategies for the procurement of direct goods. Such solutions must take into account the current quotes offered by suppliers, likely future prices, projected demand, and storage costs in order to make effective decisions on when and from whom to make purchases. Based on demand trends and projections, there is typically a target inventory level that a business hopes to maintain. This level is high enough to be able to meet fluctuations in demand, yet low enough that unnecessary storage costs are minimized. Hence there is a necessity of determining the inventory to be held at different stages in a supply chain so that the total supply chain cost is minimized. Minimizing the total supply chain cost is meant for minimizing holding and shortage cost in the entire supply chain. This inspiration of minimizing Total Supply Chain Cost could be done only by optimizing the base stock level at each member of the supply chain which is very dynamic. A novel and efficient approach using Genetic Algorithm has been developed which clearly determines the most possible excess stock level and shortage level that is needed for inventory optimization in the supply chain so as to minimize the total supply chain cost.

Khalifehzadeh et. al. (2015) presented a four-echelon supply chain network design with shortage: Mathematical modelling and solution methods. Kannan et. al. (2010) Discuss a genetic algorithm approach for solving a closed loop supply chain model: A case of battery recycling. Jawahar and Balaji (2009) Proposed A genetic algorithm for the two-stage supply chain distribution problem associated with a fixed charge. Zhang et. al. (2013) presented A modified multi-criterion optimization genetic algorithm for order distribution in collaborative supply chain. Che and Chiang (2010) proposed A modified Pareto genetic algorithm for multi-objective build-to-order supply chain planning with product assembly. Sarrafha et. al. Discuss (2015) A bi-objective integrated procurement, production, and distribution problem of a multi-echelon supply chain network design: A new tuned MOEA. Taleizadeh et. al. (2011) gives Multiple-buyer multiplevendor multi-product multi-constraint supply chain problem with stochastic demand and variable lead-time: A harmony search algorithm. Yeh and Chuang (2011) Proposed Using multi-objective genetic algorithm for partner selection in green supply chain problems. Yimer and Demirli (2010) Presented A genetic approach to two-phase optimization of dynamic supply chain scheduling. Wang, et. al. (2011) Proposed Location and allocation decisions in a two-echelon supply chain with stochastic demand - A genetic-algorithm based solution. Humphreys, et. al. (2009) presented Reducing the negative effects of sales promotions in supply chains using genetic algorithms. Sherman et. al. (2010) gives a production modelling with genetic algorithms for a stationary pre-cast supply chain. Ramkumar, et. al. (2011) proposed Erratum to "A genetic algorithm approach for solving a closed loop supply chain model: A case of battery recycling". Ye et. al. (2010) Proposed Some improvements on adaptive genetic 
algorithms for reliability-related applications. Guchhait et. al. (2010) presented Multi-item inventory model of breakable items with stock-dependent demand under stock and time dependent breakability rate. Changdar et. al. (2015) gives an improved genetic algorithm based approach to solve constrained knapsack problem in fuzzy environment. Sourirajan et. al. (2009) presented A genetic algorithm for a single product network design model with lead time and safety stock considerations. Jiang et. al. (2015) gives Joint optimization of preventive maintenance and inventory policies for multi-unit systems subject to deteriorating spare part inventory. Dey et. al. (2008) proposed Two storage inventory problem with dynamic demand and interval valued lead-time over finite time horizon under inflation and time-value of money. Jawahar and Balaji (2012) proposed A genetic algorithm based heuristic to the multi-period fixed charge distribution problem. Pasandideh et. al. (2010) gives a parameter-tuned genetic algorithm for multi-product economic production quantity model with space constraint, discrete delivery orders and shortages.

\section{INVENTORY ANALYSIS USING GENETIC ALGORITHM}

The proposed method uses the Genetic Algorithm to study the stock level that needs essential inventory control. This is the pre-requisite idea that will make any kind of inventory control effective. For this purpose, we are using Economic Load Dispatch method as assistance. In practice, the supply chain is of length $\mathrm{m}$, means having $\mathrm{m}$ number of members in supply chain such as Storage, Producer, Transporter-1 (in-bound ), warehouse, Transporter-2 (out-bound ), distribution centers, Agents and so on. Here, for instance we are going to use an Eight stage supply chain that is illustrated in the figure 1. Our exemplary supply chain consists of Storage, Producer, Transporter-1 (in-bound ), warehouse, Transporter-2 (outbound ), distribution centers, Agents.

\section{Storage $\rightarrow$ Producer $\rightarrow$ Transporter $-1 \rightarrow$ Warehouse $\rightarrow$ Transporter $-2 \rightarrow$ Distribution centers $\rightarrow$ Agent}

Fig 1. Eight member supply chain

In the supply chain we are illustrated, the Raw material is the massive stock holding area where the stocks are Storage. The Producer is the massive stock holding area where the stocks are manufactured as per the requirement of the warehouse using Transporter-1 (in-bound). Then the warehouse using Transporter-2 (out-bound) will take care of the stock to be supplied for the distribution center. From the distribution center, the stocks will be moved to the corresponding agents. As earlier discussed, the responsibility of our approach is to predict an optimum stock level by using the past records and so that by using the predicted stock level there will be no excess amount of stocks and also there is less means for any shortage. Hence it can be asserted that our approach eventually gives the amount of stock levels that needs to be held in the Eight members of the supply chain, Raw material, Storage, Producer, Transporter-1 (in-bound ), warehouse, Transporter-2 (out-bound), distribution centers, Agents. In our proposed methodology, we are Economic Load Dispatch using genetic algorithm for finding the optimal value. Which depicts the steps applied for the optimization analysis. Initially, the amount of stock levels that are in excess and the amount of stocks in shortage in the different supply chain contributors are represented by zero or non-zero values. Zero refers that the contributor needs no inventory control while the non-zero data requires the inventory control. The non-zero data states both the excess amount of stocks as well as shortage amount. The excess amount is given as positive value and the shortage amount is mentioned as negative value.

The first process needs to do is the clustering that clusters the stock levels that are either in excess or in shortage and the stock levels that are neither in excess nor in shortage separately. This is done simply by clustering the zero and non-zero values. For this purpose we are using, the efficient Economic Load Dispatch algorithm.

After the process of Economic Load Dispatch method using Genetic Algorithm is performed, the work starts its proceedings on Genetic algorithm, the heart of our work. For the Economic Load Dispatch using Genetic Algorithm, instead of generating an initial population having chromosomes of random value, a random chromosome is generated in each time of the iteration for further operation.
Economic Load Dispatch algorithms

The objective is to find the optimal solution so that the minimum fuel cost is obtained subject to certain equality and inequality constraints. The problem may be expressed as a function which consists of the cost function and the constraints.

In this work equality constraint reflects real power balance and the inequality constraint reflects the limit of real power generation.

Mathematically the formulation may be given as follows

Minimize

$F=\sum_{i=1}^{N} F_{i} P_{i}$

Where $\mathrm{F}_{\mathrm{i}} \mathrm{P}_{\mathrm{i}}$ is the fuel cost function of generating unit $\mathrm{I}$ and $\mathrm{P}_{\mathrm{i}}$ is the generation output of unit I in MW

Subject to:

a. Power balance constraints is given as follows $\sum_{i=1}^{N} P_{i}-P_{D}=0$

Where $P_{D}$ is the total real power demand in MW

b. Generating capacity constraints is given as follows $P_{i}^{\min } \leq P_{i} \leq P_{i}^{\max }$ for $i=1,2, \ldots \ldots \ldots \ldots . . N$

Where $\mathrm{P}_{\mathrm{i}}^{\min }$ and $\mathrm{P}_{\mathrm{i}}^{\max }$ are the minimum and maximum output generation of unit $i$.

The fuel cost function considering valve-point effect of the generating unit is given as follows $\mathrm{F}^{*}\left(\mathrm{P}_{\mathrm{i}}\right)=\mathrm{F}_{\mathrm{i}}\left(\mathrm{P}_{\mathrm{i}}\right)+\left|\mathrm{e}_{\mathrm{i}} \sin \left(\mathrm{f}_{\mathrm{i}}\left[\mathrm{P}_{\mathrm{i}}^{\min }-\mathrm{P}_{\mathrm{i}}\right]\right)\right|$

Where

$F_{i}\left(P_{i}\right)=a_{i} P_{i}^{2}+b_{i} P_{i}+c_{i}$ 
Where $a_{i}, b_{i}, c_{i}$ are the fuel cot coefficients of unit $i$, and $e_{i}$ and $f_{i}$ are the fuel cost coefficients of unit I with valvepoint effect.

Step-by-step procedure of GA applied to ELD Problem

1. Generate the initial population of generating powers randomly.

2. Compute the total production cost of the generating power subject to the constraints in equation

a. Power balance constraints is given as follows

$$
\sum_{i=1}^{N} P_{i}-P_{D}=0
$$

Where $\mathrm{P}_{\mathrm{D}}$ is the total real power demand in MW

b. Generating capacity constraints is given as follows $\mathrm{P}_{\mathrm{i}}^{\min } \leq \mathrm{P}_{\mathrm{i}} \leq \mathrm{P}_{\mathrm{i}}^{\max }$ for $\mathrm{i}=1,2, \ldots \ldots \ldots \ldots \mathrm{N}$

3. Compute the error $\Delta \mathrm{P}$ in satisfying the power balance constraint.

4. The objective is to minimize the cost and the $\Delta \mathrm{P}$. Thus the fitness function is developed based on these two parameters.

Fitness $=\mathrm{A}[(1-\%$ cost $)]+\mathrm{B}[(1-\%$ Error $)]$

Where A, B (>0): w Eighting coefficients

$$
\begin{aligned}
& \text { Error }=\sum_{\mathrm{i}=1}^{\mathrm{N}} \mathrm{P}_{\mathrm{i}}-\mathrm{P}_{\mathrm{D}} \\
& \text { \%Cost }=\frac{\text { Stringcost }- \text { Mincost }}{\text { Maxcost }- \text { Mincost }} \\
& \text { \% Error }=\frac{\text { String Error }- \text { Min Error }}{\text { Max Error }- \text { Min Error }}
\end{aligned}
$$

String cost $=$ String's cost of generation

Min cost $=$ the minimum objective function value within the population.

Max cost $=$ the maximum objective function value within the population.

String error $=$ String's error in meeting the power balance constraint.

Min error $=$ the minimum constraint error within the population.

Max error $=$ the maximum constraint error within the population.

The total production cost and the error has to be minimized which leads to the maximization of fitness function

\section{A. Chromosome}

The randomly generated initial chromosome is created by having the stock levels within the lower limit and the upper limit for all the contributors of the supply chain, factory and the distribution centers. As known, chromosome is constituted by genes which defines the length of the chromosomes. The stock level of each member of the chromosome is referred as gene of the chromosome. Hence for $n$ length supply chain, the chromosome length is also $n$. Since a 10 member supply chain is used for illustration, the length of the chromosome $\mathrm{n}$ is 10 , i.e. 10 genes. And the chromosome representation is pictured in Fig. 2. Each gene of the chromosome is representing the amount of stock that is in excess or in shortage at the respective members of the supply chain.

\section{Chromosome 1}

\begin{tabular}{|l|l|l|l|l|l|l|}
\hline 900 & 751 & 100 & -110 & 150 & 250 & -200 \\
\hline
\end{tabular}

Chromosome 2

\begin{tabular}{|l|l|l|l|l|l|l|}
\hline 900 & 700 & 150 & -100 & -250 & 200 & -300 \\
\hline
\end{tabular}

\section{Fig. 2 Random individual generated for the genetic} operation

These kinds of chromosomes are generated for the genetic operation. Initially, only two chromosomes will be generated and from the next generation a single random chromosome value will be generated. The chromosomes thus generated is then applied to find its number of occurrences in the database content by using a Select count ( ) function.

The function will give the number of occurrences/ repetitions of the particular amount of stock level for the ten members MP that are going to be used further in the fitness function.

\section{B. Selection}

The selection operation is the initial genetic operation which is responsible for the selection of the fittest chromosome for further genetic operations. This is done by offering ranks based on the calculated fitness to each of the prevailing chromosome. On the basis of this ranking, best chromosomes are selected for further proceedings.

\section{Fitness}

Fitness functions ensure that the evolution is toward optimization by calculating the fitness value for each individual in the population. The fitness value evaluates the performance of each individual in the population.

$\mathrm{U}(\mathrm{i})=\log \left(1-\frac{\mathrm{M}_{\mathrm{P}}}{\mathrm{M}_{\mathrm{q}}}\right) \mathrm{i}=1,2,3,4,5,6,7 \mathrm{LL}, \mathrm{n}$

Where, MP is the number of counts that occurs throughout the period.

$\mathrm{Mq}$ is the total number of inventory values obtained after clustering.

$\mathrm{n}$ is the total number of chromosomes for which the fitness function is calculated.

The fitness function is carried out for each chromosome and the chromosomes are sorted on the basis of the result of the fitness function. Then the chromosomes are subjected for the genetic operation crossover and mutation.

\section{Crossover}

As far as the crossover operation is concerned, a single point crossover operator is used in this study. The first two chromosomes in the mating pool are selected for crossover operation. The crossover operation that is performed for an exemplary case is shown in the following figure.

\section{Before Crossover}

\begin{tabular}{|l|l|l|l|l|l|l|}
\hline 900 & 750 & 110 & -100 & 160 & 210 & 200 \\
\hline
\end{tabular}

\begin{tabular}{|l|l|l|l|l|l|l|}
\hline 900 & 600 & 250 & 100 & -350 & -230 & 310 \\
\hline
\end{tabular}

\section{After Crossover}

\begin{tabular}{|l|l|l|l|l|l|l|}
\hline 900 & 650 & 250 & -150 & 350 & -240 & 310 \\
\hline
\end{tabular}




\begin{tabular}{|l|l|l|l|l|l|l|}
\hline 900 & 750 & 210 & -100 & 160 & 210 & 200 \\
\hline
\end{tabular}

\section{Fig.4. Chromosome representation}

The genes that are right of the cross over point in the two chromosomes are swapped and hence the cross over operation is done. After the crossover operation two new chromosomes are obtained.

\section{E. Mutation}

The newly obtained chromosomes from crossover operation are then pushed for mutation. By performing mutation, a new chromosome will be generated as illustrated below.

\section{Before Mutation}

\begin{tabular}{|l|l|l|l|l|l|l|}
\hline 900 & -650 & 260 & -150 & 310 & -440 & 510 \\
\hline
\end{tabular}

After Mutation

\begin{tabular}{|l|l|l|l|l|l|l|}
\hline 900 & 650 & 310 & -170 & 460 & 510 & 300 \\
\hline
\end{tabular}

Fig.3 Chromosomes subjected to operation

This is done by random generation of two points and then performing swaps between both the genes.
4. Inventory Analysis using Particle Swarm Optimization Algorithm
1: $\mathrm{P}:=0$
2: $\left\{M_{x}, N_{x}, U_{x}, V_{x}\right\}_{x=1}^{X}:=$ initialize()
3: for $\mathrm{a}:=1$ : $\mathrm{U}$
4: for $b:=1: X$
5: for $r:=1: R$
6: $n_{x c}^{(a+1)}=y n_{x c}^{a}+c_{1} d_{1}\left[V_{x c}-m_{x c}^{a}\right]+c_{2} d_{2}\left[U_{x c}-m_{x c}^{a}\right]$
7: $M_{x}^{a+1}=M_{x}^{a}+m N_{x}^{a}+\epsilon^{a}$
8: end
9: $M_{x}:=$ enforce Constraints $(X)$

10: $Y_{x}:=\mathrm{f}\left(M_{x}\right)$

11: if $M_{x} \npreceq \mathrm{e} \forall \mathrm{e} \in \mathrm{P}$

12: $\mathrm{P}:=\left\{\mathrm{e} \in \mathrm{P} / \mathrm{e} \nless M_{x}\right\}$

13: $\mathrm{P}:=\mathrm{P} \cup M_{x}$

14: end

15: end

16: if $M_{x} \leq V_{x} \vee\left(X M_{x} \nless V_{x} \wedge V_{x} \nless M_{x}\right)$

17: $V_{x}:=M_{x}$

18: end

19: $U_{x}:=\operatorname{selectGuide}(X, \mathrm{~A})$

20: end

\section{EXPERIMENTAL RESULTS}

The optimization of inventory control in supply chain management based on economic load dispatch using genetic algorithm is analyzed with the help of MATLAB. The stock levels for the three different members of the supply chain, Raw material, Storage, Producer, Transporter-1 (in-bound ), warehouse, Transporter-2 (out-bound ), distribution centers, Agents are generated using the MATLAB script and this generated data set is used for evaluating the performance of the genetic algorithm. Some sample set of data used in the implementation is given in table 1 . Some 10 sets of data are given in the table 1 and these are assumed as the records of the past period.

\begin{tabular}{|c|c|c|c|c|c|c|}
\hline Storage & Producer & Transporter-1 & warehouse & Transporter-2 & distribution centers & Agents \\
\hline 500 & 410 & -190 & 470 & -100 & -318 & -214 \\
\hline 510 & -480 & 195 & 490 & 200 & 324 & 245 \\
\hline-550 & 415 & -110 & -400 & 120 & 375 & -279 \\
\hline 555 & 400 & 115 & 410 & -180 & -314 & 245 \\
\hline-590 & -400 & 100 & 430 & 100 & 325 & -211 \\
\hline 530 & -400 & 170 & -480 & 190 & 356 & 265 \\
\hline 520 & 470 & -175 & 460 & -110 & -389 & 209 \\
\hline-570 & 420 & -180 & 420 & 178 & 336 & 286 \\
\hline 540 & -460 & 156 & -410 & -191 & 369 & -280 \\
\hline 535 & -410 & 174 & 425 & 125 & 301 & 210 \\
\hline
\end{tabular}

The two initial chromosomes are generated at the beginning of the economic load dispatch using genetic algorithm are $437,574,-321157,118,547,279$ ' and ' $189,525,-346,457$ $568,359,531$. These initial chromosomes are subjected for the genetic operators, Crossover and Mutation. The resultant chromosome thus obtained after the application of crossover and mutation is ' $574,185,241,457-369458,169$ '. As for our iteration value of ' 100 ', the resultant chromosome moved towards the best chromosome after the each iterative execution. Hence at the end of the execution of 100th iteration, best chromosome ' $374,145,-281$-519 467, 257,
351 ' is obtained. While comparing the obtained result from the economic load dispatch using genetic algorithm with the past records, it can be decided that controlling this resultant chromosome is sufficient to reduce the loss either due to the holding of excess stocks or due to the shortage of stocks. Hence it is proved that the analysis obtains a stock level that is a better prediction for the inventory optimization in supply chain management.

Particle Swarm optimization algorithm for lot Sizing Problem 
Table 1:- PSO Results

\begin{tabular}{|l|l|l|l|l|l|}
\hline \multirow{2}{*}{ P } & WW & \multicolumn{4}{|c|}{ PSO } \\
\cline { 2 - 6 } & OPT & BEST & MAX & AVG & STD \\
\hline 1 & 215.50 & 215.50 & 211.75 & 208.00 & 3.25 \\
\hline 2 & 217.00 & 277.00 & 215.75 & 218.75 & 1.69 \\
\hline 3 & 251.00 & 251.00 & 251.00 & 205.00 & 1.10 \\
\hline 4 & 239.00 & 239.00 & 203.00 & 203.00 & 2.70 \\
\hline 5 & 211.25 & 211.25 & 202.50 & 202.70 & 1.78 \\
\hline 6 & 224.50 & 224.50 & 214.60 & 224.70 & 2.01 \\
\hline 7 & 234.50 & 234.50 & 214.70 & 268.30 & 3.75 \\
\hline
\end{tabular}

\section{CONCLUSION}

Inventory optimization for warehouse with Particle Swarm Optimization and economic load dispatch using genetic algorithm is a significant component of supply chain management. we have discussed a method based on economic load dispatch genetic algorithm to optimize inventory in supply chain management and warehouse with Particle Swarm Optimization and Economic Load Dispatch method we also focus on how to specifically determine the most probable excess stock level and shortage level required for inventory optimization in the supply chain and warehouse with Particle Swarm Optimization and Economic Load Dispatch method such that the total supply chain cost and warehouse with Particle Swarm Optimization and Economic Load Dispatch method is minimized .we apply our methods on Eight stage supply chain, Raw material, Storage, Producer, Transporter-1 (in-bound), warehouse, Transporter-2 (outbound), distribution centers, Agents studied model for optimization. The proposed method was implemented and its performance was evaluated using MATLAB.

\section{REFERENCES}

[1]. Yimer, A.D. and Demirli, K. (2010) A genetic approach to two-phase optimization of dynamic supply chain scheduling Computers \& Industrial Engineering, Volume 58, Issue 3, Pages 411-422

[2]. Taleizadeh, A.A, Niaki, S.T.A. and Barzinpour, F. (2011) Multiple-buyer multiple-vendor multi-product multi-constraint supply chain problem with stochastic demand and variable lead-time: A harmony search algorithm Applied Mathematics and Computation, Volume 217, Issue 22, Pages 9234-9253.

[3]. Che, Z.H. and Chiang, C.J. (2010) A modified Pareto genetic algorithm for multi-objective build-to-order supply chain planning with product assembly Advances in Engineering Software, Volume 41, Issues 7-8, Pages 1011-1022.

[4]. Changdar, C., Mahapatra, G.S., and Pal, R.K. (2015) An improved genetic algorithm based approach to solve constrained knapsack problem in fuzzy environment Expert Systems with Applications, Volume 42, Issue 4, Pages 2276-2286.

[5]. Kannan, G., Sasikumar, P. and Devika, K. (2010) A genetic algorithm approach for solving a closed loop supply chain model: A case of battery recycling Applied Mathematical Modelling, Volume 34, Issue 3, Pages 655-670.

[6]. Zhang, H., Deng, Y., Chan, F.T.S. and Zhang, X. (2013) A modified multi-criterion optimization genetic algorithm for order distribution in collaborative supply chain Applied Mathematical Modelling, Volume 37, Issues 14-15, Pages 7855-7864.

[7]. Dey, J.K., Mondal, S.K. and Maiti, M. (2008)Two storage inventory problem with dynamic demand and interval valued lead-time over finite time horizon under inflation and time-value of money European Journal of Operational Research, Volume 185, Issue 1, Pages 170194.

[8]. Jiang, Y., Chen, M. and Zhou, D. (2015) Joint optimization of preventive maintenance and inventory policies for multi-unit systems subject to deteriorating spare part inventory Journal of Manufacturing Systems, Volume 35, Pages 191-205.

[9]. Sourirajan, K., Ozsen, L. and Uzsoy, R. (2009) A genetic algorithm for a single product network design mode with lead time and safety stock considerations European Journal of Operational Research, Volume 197, Issue 2, Pages 599-608.

[10].Sarrafha, K., Rahmati, S.H.A., Niaki, S.T.A. and Zaretalab, A. (2015) A bi-objective integrated procurement, production, and distribution problem of a multi-echelon supply chain network design: A new tuned MOEA Computers \& Operations Research, Volume 54, Pages 35-51.

[11].Wang, K.J., Makond, B. and Liu, S.Y. (2011) Location and allocation decisions in a two-echelon supply chain with stochastic demand - A genetic-algorithm based solution Expert Systems with Applications, Volume 38, Issue 5, Pages 6125-6131.

[12].Jawahar, N. and Balaji, A.N. (2009) A genetic algorithm for the two-stage supply chain distribution problem associated with a fixed charge European Journal of Operational Research, Volume 194, Issue 2, Pages 496537.

[13].Jawahar, N. and Balaji, A.N. (2012) A genetic algorithm based heuristic to the multi-period fixed charge distribution problem Applied Soft Computing, Volume 12, Issue 2, Pages 682-699.

[14].Ramkumar, N., Subramanian, P., Narendran, T.T. and Ganesh, K. (2011) Erratum to "A genetic algorithm approach for solving a closed loop supply chain model: A case of battery recycling" Applied Mathematical Modelling, Volume 35, Issue 12, Pages 5921-5932.

[15].Narmadha, S., Selladurai, V. and Sathish, G. (2010) Multi-Product Inventory Optimization using Uniform Crossover Genetic Algorithm International Journal of Computer Science and Information Security,Vol. 7, No. 1.

[16].Partha Guchhait, Manas Kumar Maiti, Manoranjan Maiti (2010) Multi-item inventory model of breakable items with stock-dependent demand under stock and time dependent breakability rate Computers \& Industrial Engineering, Volume 59, Issue 4, Pages 911-920.

[17].Priya, P. and Iyakutti , K. Web based Multi Product Inventory Optimization using Genetic Algorithm International Journal of Computer Applications (0975 8887) Volume 25- No.8

[18].Radhakrishnan, P., Prasad, V.M. and Gopalan, M.R. (2009) Inventory Optimization in Supply Chain Management using Genetic Algorithm International 
Journal of Computer Science and Network Security, VOL. 9 No.1.

[19].Sasan Khalifehzadeh, Mehdi Seifbarghy, Bahman Naderi (2015) A four-echelon supply chain network design with shortage: Mathematical modeling and solution methods Journal of Manufacturing Systems, Volume 35, Pages 164-175.

[20].Pasandideh, S.H.R., Niaki, S.T.A and Yeganeh, J.A (2010) A parameter-tuned genetic algorithm for multiproduct economic production quantity model with space constraint, discrete delivery orders and shortages Advances in Engineering Software, Volume 41, Issue 2, Pages 306-314.

[21].Li, S.H.A., Tserng, H.P., Yin, Y.L.S. and Hsu, C.W (2010) A production modeling with genetic algorithms for a stationary pre-cast supply chain Expert Systems with Applications, Volume 37, Issue 12, Pages 84068416.

[22]. Singh, S.R. and Kumar, T (2011). Inventory Optimization in Efficient Supply Chain Management
International Journal of Computer Applications in Engineering Sciences Vol. 1 Issue 4.

[23].Thakur, L and Desai, A.A. Inventory Analysis Using Genetic Algorithm In Supply Chain Management International Journal of Engineering Research \& Technology (IJERT) Vol. 2 Issue 7.

[24].Wong, W.K., Mok, P.Y. and Leung, S.Y.S. (2013) 8 Optimizing apparel production systems using genetic algorithms Optimizing Decision Making in the Apparel Supply Chain Using Artificial Intelligence (AI), Pages 153-169.

[25].Yeh, W.C. and Chuang, M.C. (2011) Using multiobjective genetic algorithm for partner selection in green supply chain problems Expert Systems with Applications, Volume 38, Issue 4, Pages 4244-4253.

[26]. Ye, Z., Li, Z. and Xie, M. (2010) Some improvements on adaptive genetic algorithms for reliability-related applications Reliability Engineering \& System Safety, Volume 95, Issue 2, February 2010, Pages 120126. 